\title{
Ocupación por arboles de calidad: un concepto para evaluar plantaciones
}

\author{
María Martha Rodríguez Arellano ${ }^{1}$ \\ Martín Alfonso Mendoza Briseño ${ }^{1}$ \\ Carlos Héctor Avila Bello ${ }^{1}$ \\ José Evaristo Pacheco Velazco ${ }^{1}$
}

\section{RESUMEN}

Se propone el concepto de ocupación por árboles de calidad como un medio para evaluar plantaciones. Este concepto aplica la noción de parcela vacía sobre una escala de vitalidad que va desde muerto hasta individuo excelente. La frecuencia de cada calidad de individuo a su vez se ajusta a un modelo de regresión sobre los factores causales del ambiente y los patrones de desarrollo del árbol. Un ejemplo con plantaciones de especies tropicales en Los Tuxtlas, Veracruz, México, ilustra que al aplicar esta técnica se obtiene una representación cuantitativa del estado de la plantación y de esta representación se deducen causas probables, se estima el desarrollo inmediato y se sugiere la prescripción de labores correctivas que pudieran requerirse.

PALABRAS CLAVE:

Evaluación de plantaciones, ocupación de sitio, calidad de arbolado, plantaciones tropicales, establecimiento de brinzales.

\begin{abstract}
The quality tree occupancy concept is presented as a basis for evaluation of plantations. This concept joins the null plot idea with a vitality rating that goes from a dead tree to an excellent tree. Quality frequencies are then fitted to explanatory variables that represent environment, and tree development patterns. A sample of tropical plantations from Los Tuxtlas, Veracruz, Mexico, serves as an example illustrating the concept's capability to depict quantitatively a plantation condition. The model fitted to the Tuxtlas example demonstrated that it is possible to infer a biological interpretation of current conditions, estimate a prognosis of further development, and suggest prescriptions to correct trends, should the need arose.
\end{abstract}

KEY WORDS:

Plantation evaluation, site occupancy, stock quality, tropical plantations, seedling establishment. 


\section{INTRODUCCION}

El desarrollo temprano de plantaciones ha sido motivo de constante interés de investigación por la necesidad de anticipar tendencias que puedan corregirse con técnicas silvícolas. El conocimiento sobre técnicas de manejo, respuesta a labores culturales, economía e influencias ambientales ha crecido enormemente en últimos años (Clifton 1985, Maugé 1987, James y Tarlton 1990). En el tema de evaluación silvícola gran parte de la atención se ha centrado en estimar el número de sobrevivientes a cierta edad, por unidad de superficie, complementado por estimaciones de crecimiento en altura (por ejemplo, Mas et al 1990, Chacón y Manzanilla 1988). Sin embargo estos enfoques cuantitativos no nos dan idea clara del desempeño biológico de las plantaciones y menos de su problemática silvícola.

El problema de evaluación en el trópico merece atención especial porque el carácter de las plantaciones no es forestal, pues son pequeñas superficies aisladas, sin una intención de soportar a $n$ sistema industrial de aprovechamiento; por ello no hay forma de financiar estudios a detalle. Además, los hábitos y la forma de las especies tropicales no se prestan del todo al manejo cuantitativo usual en evaluaciones en zonas templadas. Sin embargo, entidades importantes en reforestación tropical, como el gobierno del Estado de Veracruz, insisten en usarlos esquemas tradicionales de evaluación.

Lo incipiente de los actuales elementos de evaluación temprana de plantaciones es justificable a la luz de las dificultades estadísticas para establecer si suficientes individuos desarrollarán exitosamente, cuando en las primeras dos a cinco temporadas de crecimiento en campo se muestran tan aparentemente homogéneos y aun sin sufrir los efectos del tiempo y los factores ecológicos restrictivos. Por otra parte, esperar más de cinco años para detectar diferencias en desempeño facilitaría la labor estadística pero es silvícolamente inaceptable, pues si hubiere consecuencias severas ya no podrán corregirse.

En silvicultura existe consenso acerca de la importancia de dos conceptos: ocupación de sitio y árbol de calidad. El primero se refiere a que los individuos plantados estén en número suficiente y distribuidos a manera de no dejar espacios productivos sin ocupar (Clutter et al. 1983, Oliver y Larson 1996). El segundo concepto, el de calidad de árbol, implica que si el individuo no llena ciertas especificaciones administrativamente impuestas, y que derivan de los objetivos de la plantación, su espacio ocupado se registrará en la evaluación como si estuviese vacío. Concurrentemente, si en un punto a evaluar se localiza más de un árbol de calidad, sólo se contabiliza uno de ellos, en el entendido que los sobrantes serán removidos en el curso de las labores de control de densidad (Stein 1978).

La unión de estos dos conceptos, denotada como ocupación de árboles de calidad, crea la posibilidad de estimar la eficiencia en ocupación real del espacio de crecimiento. Este trabajo propone una forma de evaluar plantaciones en la que la ocupación de los árboles de calidad se asocie estadísticamente con los factores explicativos o causales del desempeño de la plantación. El fin perseguido es obtener estimadores de desempeño que tengan la posibilidad de ser interpretados silvícolamente y de sugerir acciones a tomar para el manejo posterior de la plantación. El uso de un ejemplo práctico ilustrará la aplicación del concepto y clarificará los elementos de procedimiento. La discusión subsecuente versará sobre la eficacia de este nuevo concepto a partir de las peculiaridades del ejemplo usado. 


\section{MATERIALES Y METODOS}

Para ejemplo se escogieron 16 plantaciones de especies preciosas tropicales de entre un gran número de otras existentes en Veracruz, México. Estas plantaciones son las más diversas encontradas dentro de las que se consideraron representativas de las técnicas usadas por los programas de reforestación en vigor desde 1989. Debido a lo reciente de las plantaciones, la muestra tiende a conformarse de aquellas de mayor edad, en este caso de 2 a 6 temporadas de crecimiento en campo. La intención general es captar aquel estado en que ya se tienen efectos mesurables, pero no han llegado los daños a un grado que no se puedan atender las causas al través de labores de cultivo. Los datos de archivo de la Secretaría de Agricultura, Ganadería y Desarrollo Rural, y la información verbal de los responsables permitieron describir el régimen de manejo y garantizar su homogeneidad y comparabilidad.Las plantaciones muestreadas contienen cedro (Cedrela odorata L.) primavera (Roseodendron donel-smithii Rose y Miranda), caoba (Swietenia macrophylla King), y roble (Tabebuia rosea Bertol). Ellas se encuentran dentro de un radio de $20 \mathrm{Km}$ alrededor de Catemaco, Veracruz, entre los

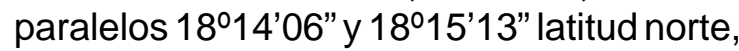
y 9500' 85 " y 9515'04" longitud oeste, en la región de Los Tuxtlas. El terreno es montañoso con gradientes altitudinales de cero a 1700 msnm (Souza citado por Dirzo y García, 1992). El clima es cálido húmedo con abundantes lluvias en verano, temperatura promedio de $25^{\circ} \mathrm{C}$ y precipitación media anual de $2500 \mathrm{~mm}$ (García, 1988). Los feozems son los suelos predominantes, tienen fases pedregosas y no son muy permeables. La vegetación original era selva alta perennifolia, de la cual quedan escasos relictos. En el uso del suelo predominan la agricultura y la ganadería. Hay antecedentes que dicen que los terrenos plantados previamente se usaron para actividades agropecuarias, además de que en la actualidad están rodeados por cultivos y potreros. O sea que tanto el ambiente actual como el resultado final buscado distan de ser un bosque.

Como las plantaciones tropicales suelen ser minúsculas, en la selección de plantaciones muestra se exigió que éstas fuesen mayores de $0.5 \mathrm{Ha}$. Cada una de ellas se buscó de aspecto homogéneo, excepto cuando por causas evidentes y conocidas hubiere dos condiciones distintas, pues entonces cada condición representa un caso distinto. Las plantaciones en la muestra ocupan de 05 a $3.5 \mathrm{Ha}$.

La unidad muestral es un punto al azar dentro de la plantación, habiendo colectado 20 de ellos en cada caso. El procedimiento para el sorteo consistió en usar parejas de números aleatorios y convertirlos en coordenadas a la escala que corresponda para la extensión de la plantación, localizándolos con brújula y longímetro.

A partir de cada punto se midieron las distancias al árbol más cercano, al segundo más cercano, y desde el primero a su vecino más cercano (Mueller-Dumbois y Ellenberg, 1974). La primera se tomó como el radio de un círculo de terreno desocupado, o parcela vacía (Valdez, 1992). Con estos datos se construyeron distribuciones de frecuencias de espacio vacío en función de su tamaño.

Entendiendo que el desempeño de la plantación se expresa como sobre vivencia y vitalidad del arbolado, ambas se consideraron en una sola variable denotada VITAL. Esta variable es una evaluación subjetiva global del individuo y tiene 7 niveles, de excelente (6) a pésimo (1), con muerto indicado por cero.

La primera parte del procesamiento involucra comparar las distribuciones de frecuencias de las tres distancias. Para ello se recurrió a la prueba de $t$ sobre las diferencias entre las medias de las distancias, previa verificación de la 
homogeneidad de varianzas. Este procedimiento es equiparable a los índices de contagio con que usualmente se prueba si la distribución de plantas en el terreno es al azar, homogénea o en conglomerados (Müeller-Dumbois y Ellenberg, 1974).

Para explicar la variable de respuesta se tomaron datos del sitio, del individuo y del historial de eventualidades y labores de cultivo (Tabla1). En esta lista predominan variables subjetivamente definidas, y ello es en parte con la intención de probar si métodos expeditos fueran suficientes para detectar el desempeño de los árboles, pero principalmente por la falta de información publicada sobre especies tropicales, y ante el riesgo de trasladar al ámbito tropical las controversias de la definición de calidad que predominan en ambientes templados (Puttonen, 1997; Bayley y Kietzka, 1997). En el evento de detectar relaciones fuertes de los factores considerados, este estudio de carácter exploratorio puede dar lugar a investigaciones subsecuentes que substituyan las variables subjetivas por estimaciones cuantitativas de los mismos factores.

En una primera depuración para detectar a los mejores factores predictivos de VITAL, se construyeron relaciones lineales entre las variables explicadoras, transformadas y combinaciones de ellas, respecto a VITAL, desechando las variables débilmente asociadas. En la siguiente depuración, usando regresión por pasos, se seleccionaron los factores que mayor peso tienen en abatir el cuadrado medio error del modelo. Algunas variables fueron forzadas a entrar en la versión final para contar con descripciones de factores trascendentes para el manejo, sin importar que los coeficientes de estos factores fuesen pequeños, en tanto no se deteriorase el ajuste del modelo. Consideraciones similares a las descritas por Bayley y Kietzka (1997) y Bigras (1998), también influyeron en la selección de variables, lo mismo que las gráficas de dispersión de cada variable respecto a VITAL.

La elección de un modelo lineal estuvo influenciada por la falta de ajuste de modelos logísticos (logit y probit), que son los métodos usuales para modelar procesos de sobre vivencia.

\section{RESULTADOS Y DISCUSION}

Indagando sobre la homogeneidad del espaciamiento, la prueba de diferencias de medias entre distancia al cercano, al siguiente y al vecino más cercano no encontró que fuesen estadísticamente diferentes (Fig. 1), o sea, que para fines de cultivo cabe afirmar que la cobertura actual es homogénea, no hay ni aglomeraciones ni tampoco grandes huecos.

Otra evidencia más es la figura 2, que presenta la frecuencia acumulativa de huecos por clase de VITAL, desplegando consistentemente para todas las clases un patrón de rápido incremento en frecuencias hasta llegar a los $9 \mathrm{~m}$, siendo raras las instancias de espacios con radios mayores. Significa entonces que no hay grandes huecos, claro que esta deducción está sujeta a giros de interpretación debido a lo reciente de las plantaciones del ejemplo, y por el efecto de la replantación de fallas, además de que en plantaciones pequeñas no hay forma de tener grandes huecos a menos que sea falla total. La densidad promedio en el total de la muestra es de $1285 \pm 27$ individuos por hectárea. En términos silvícolas esta cifra y los datos de homogeneidad dan a entender que efectivamente la tecnología utilizada es eficaz en cuanto a cubrir suficientemente el terreno plantado, considerando que el mínimo hueco replantable fuese de $100 \mathrm{~m}^{2}$, que con las fallas durante el turno, más las labores de aclareo, den un a densidad al momento de cosecha tal que el terreno esté ocupado a capacidad. 
Tabla 1. Lista de variables consideradas.

Distancia, en cm (cercano/segundo/vecino)

Vitalidad $=7$ categorías

Edad, en número de temporadas de secas en campo

Diámetro basal, en $\mathrm{mm}$

Area basal = 61520; 61472; 61482; (diámetro/2)2

Altura fustal, en $\mathrm{cm}$

Largo de copa, en $\mathrm{cm}$

Radio de copa, en cm

Proporción de copa, largo/altura

Area de copa $=61520$; radio $^{2}$

Volumen de copa $=$ area*lago/2

Simetría de copa, 5 categorías

Frondosidad, escala 5 categorías

Color de follaje, descriptivo

Verticalidad del fuste, 5 categorías

Ahusamiento del fuste, 5 categorías

Escamas y placas de corteza en el tallo, 5 categorías

Yemas, 5 categorías

Manejo: cualquiera de las siguientes acciones: preparación de sitio intensa, control de malezas, eliminar el dosel superior, evitar el ingreso de ganado, control de combustibles, poda, cultivos asociados

Tendencia: proyección del desarrollo inmediato del individuo dada la actual distribución del espacio aéreo a su alrededor. Tres categorías: regresiva, estacionaria, progresiva

Sanidad: Suma de efectos de daños, defectos, siniestros previos, plagas y enfermedades, en 5 categorías

Cobertura del sitio \%: suelo mineral, piedra, hojarasca, hierbas, arbustos, árboles, cada uno

Suelo: tipo (carta INEGI)

Suelo color: descriptivo

Suelo daños: por erosión o compactación 5 categorías

El resultado sobre la calidad de estas plantaciones no es tan optimista como lo es el de la cobertura. En la figura 2 nótese que la mayoría de individuos pertenece a las clases de VITAL 3 y 4 . Es injustificable para una población mostrar diferenciación de copas sin aun haber cerrado el dosel (Oliver y Larson, 1996). Las observaciones colaterales en campo y los datos de cobertura de sotobosque apuntan a un papel menor de la competencia con arvenses, de donde se deduce que el pobre desempeño actual en el arbolado se debe a otra cosa, como factores de tipo genético, mala técnica en vivero y/o en la plantación. La necesidad de tomar medidas culturales es acentuada por el hecho de que las variables de tendencia apuntan a un deterioro paulatino de la situación actual. Esta tendencia a declinar es corroborada no sólo por los datos de tendencia, sino también por las calificaciones de VITAL, que empeoran para el arbolado de mayor edad en la muestra.

El modelo que mejor relacionó los factores explicatorios con la respuesta en VITAL tiene un coeficiente de determinación 


\section{Frecuencia en la muestra}

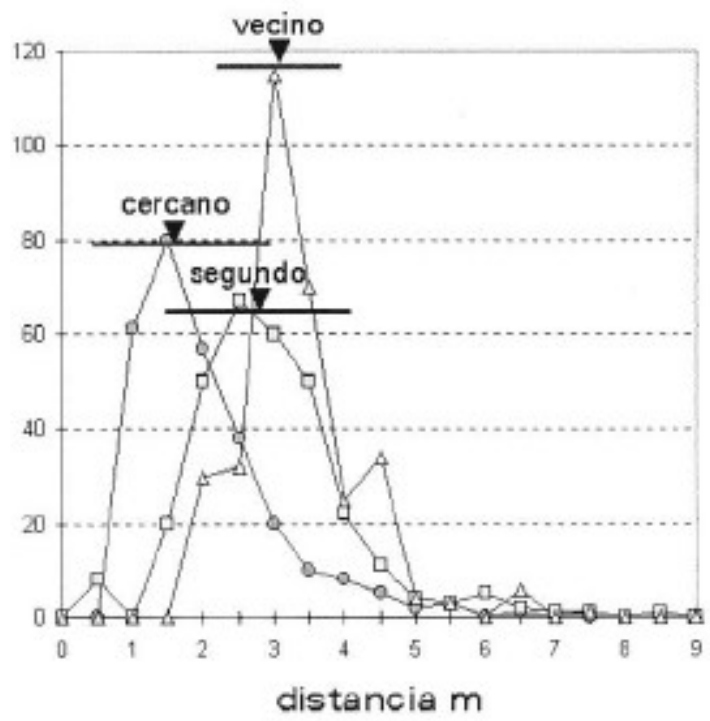

Figura 1. Media, desviación y frecuencias de distancias del punto al árbol más cercano, el segundo más cercano y entre el más cercano y su vecino.

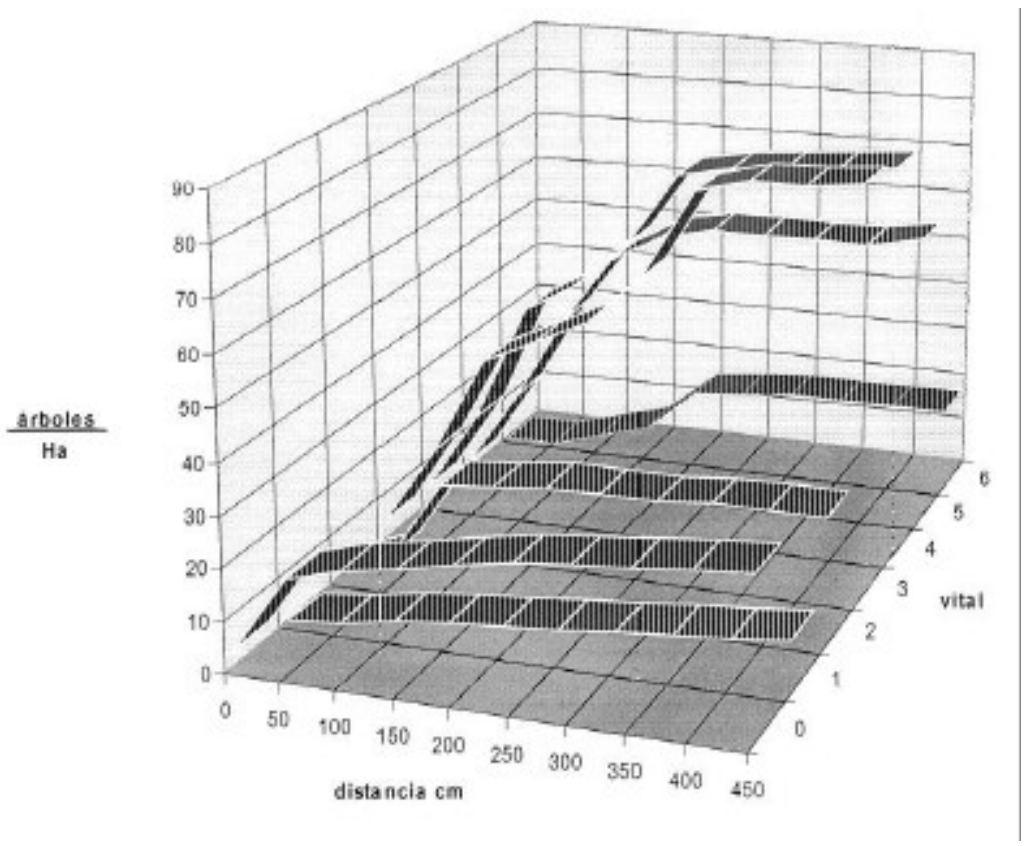

Figura 2. Distribución acumulativa de frecuencias de distancia entre el punto y el árbol más cercano, por categorías de VITAL. 
de $R^{2}=0.87$, en tanto el análisis de varianza del modelo (Tabla 2) es altamente significativo $(P(F)<0.0001)$. El modelo en cuestión es:

$$
\begin{aligned}
& \text { VITAL }=0.420758+(0.143426 * \text { INDICEB }) \\
& +\left(0.0044705^{*} \text { LCOPA }\right)+\left(0.004961^{*} \text { RCOPA }\right) \\
& \left.-0.0099322^{*} \text { PCOPA }\right)+\left(0.133638{ }^{*} \text { EDAD }\right)+e
\end{aligned}
$$

En donde:

INDICEB = Transformación lineal de forma (simetría, ahusamiento fustal, verticalidad), corteza, yemas frondosidad, sanidad, defectos y tendencia.

LCOPA = largo de copa

$\mathrm{RCOPA}=$ radio de copa

PCOPA = proporción de copa

EDAD $=$ edad
La intención del INDICEB es captar la interacción de actores no cuantitativos, luego de saber que uno por uno ninguno de ellos tiene gran peso.

Las variables sin mucho peso se dejaron para crear una representación interpretable biológicamente fueron las variables de copa. Cabe aclarar que el permitir a PCOPA un coeficiente negativo es con el fin de crear un efecto de curvatura que se dará con la interacción con RCOPA y LCOPA para las dimensiones usuales de los individuos estudiados; hay que entender que este modelo puede no tener el comportamiento deseado si las situaciones son de excepción. El modelo creado es consistente con los aspectos teóricos del desarrollo de los árboles en general, y

\begin{tabular}{|c|c|c|c|c|c|}
\hline FUENTE & GL & $\begin{array}{c}\text { SUMA DE } \\
\text { CUADRADOS }\end{array}$ & $\begin{array}{c}\text { CUADRADO } \\
\text { MEDIO }\end{array}$ & $\mathrm{F}$ & $\mathrm{PROB}>\mathrm{F}$ \\
\hline \begin{tabular}{|l} 
Modelo \\
Error 314 \\
Total 319 \\
\end{tabular} & $\begin{array}{c}5 \\
114.0 \\
906.0 \\
\end{array}$ & 792.0 & $\begin{array}{l}158.40 \\
0.36\end{array}$ & 436.3 & 0.0001 \\
\hline VARIABLE & $\mathrm{GL}$ & COEFICIENTE & $\begin{array}{c}\text { ERROR } \\
\text { ESTANDAR }\end{array}$ & $\begin{array}{c}\mathrm{T} \\
\text { PROBANDO } \\
\text { COEF. }=0\end{array}$ & $\mathrm{PROB}>*_{\mathrm{t}} *$ \\
\hline $\begin{array}{l}\text { INTERCEPT } \\
=0.420758+(0, \\
44705 * \text { LCOPA) } \\
9322{ }^{*} \text { PCOPA) } \\
\\
\text { INDICEB } \\
\text { LCOPA } \\
\text { PCOPA } \\
\text { RCOPA } \\
\text { EDAD }\end{array}$ & $\begin{array}{c}1 \\
143426 * \\
+(0.004 \\
+(0.1336 \\
\\
1 \\
1 \\
1 \\
1\end{array}$ & $\begin{array}{c}-0.420758 \\
\text { INDICEB) } \\
961^{*} \text { RCOPA) } \\
38 \text { *EDAD) +e } \\
\\
0.143425 \\
0.004705 \\
-0.009322 \\
0.004961 \\
0.133638\end{array}$ & $\begin{array}{l}0.12402 \\
0.00432 \\
0.00105 \\
0.00279 \\
0.00167 \\
0.03263\end{array}$ & $\begin{array}{c}-3.393 \\
33.184 \\
4.483 \\
-3.337 \\
2.974 \\
4.095\end{array}$ & $\begin{array}{l}0.0008 \\
0.0001 \\
0.0001 \\
0.0009 \\
0.0032 \\
0.0001\end{array}$ \\
\hline \multicolumn{2}{|c|}{ VARIABLE } & SC TIPO I & SC TIPO II & $\begin{array}{c}\text { ESTIMADA } \\
\text { ESTAND. }\end{array}$ & $\begin{array}{l}\text { INFLACION } \\
\text { VARIANZA }\end{array}$ \\
\hline \multicolumn{2}{|l|}{$\begin{array}{l}\text { INTERCEPT } \\
\text { INDICEB } \\
\text { LCOPA } \\
\text { PCOPA } \\
\text { RCOPA } \\
\text { EDAD } \\
\end{array}$} & $\begin{array}{c}3380.00 \\
763.23 \\
12.34 \\
7.69 \\
2.64 \\
6.09 \\
\end{array}$ & $\begin{array}{c}4.18 \\
399.77 \\
7.30 \\
4.04 \\
3.21 \\
6.09 \\
\end{array}$ & $\begin{array}{c}0.0000 \\
0.9244 \\
0.1434 \\
-0.1133 \\
0.0834 \\
0.0992 \\
\end{array}$ & $\begin{array}{l}0.000 \\
1.937 \\
2.554 \\
2.876 \\
1.961 \\
1.463 \\
\end{array}$ \\
\hline
\end{tabular}
tropicales en especial.

Tabla 2. Regresión y análisis de varianza del modelo de vitalidad (VITAL) 
Esta ecuación describe que la condición de vitalidad del individuo mejora con la edad y con el tamaño de copa, pero principalmente depende del cuadro de síntomas e indicios de vigor, salud y estructuras que implican potencial de desarrollo, como son la sanidad, la tendencia dinámica, la verticalidad del fuste, las yemas, las escamas de la corteza, y el follaje. Tristemente, para el ejemplo seguido las calificaciones de VITAL no son favorables y por tanto de poco sirve que se tenga tan eficiente cobertura y sobre vivencia. Evaluaciones con métodos convencionales como los usados por Mas et al. (1990), y que son similares a los que emplea la Secretaría de Agricultura y Desarrollo Rural, no hubieran podido llegar a este tipo de información y menos podrían dar luz sobre las causas y los probables remedios.

\section{CONCLUSIONES}

Se propone una forma de evaluación de plantaciones bajo el nombre de ocupación por árboles de calidad. Esta metodología es especialmente eficaz en situaciones no forestales como el caso de las numerosas plantaciones de especies preciosas tropicales veracruzanas. Aprovechando la noción de parcela vacía y relacionando ésta con factores explicativos interpretables silvícolamente se logra una representación cuantitativa del estado de la plantación tal que es posible un diagnóstico biológico de su situación y un esbozo de las medidas culturales en respuesta.

En el ejemplo sobre plantaciones en Los Tuxtlas, Veracruz, se detectó una cobertura suficiente, homogénea, libre aun de catástrofes, pero de calidad mediocre y vitalidad con tendencia a empeorar. El cuidado posterior de estas plantaciones se anticipa debe ser esmerado para detener la declinación generalizada que hoy predomina. El fracaso de los modelos logísticos para los datos el ejemplo de Los Tuxtlas no implica que este tipo de modelos puedan representar fielmente la naturaleza del proceso de establecimiento de otras plantaciones.

\section{REFERENCIAS}

Bayley, A.D. y J.W.. Kietzka. 1997. Stock quality and field performance of Pinus patula seedlings produced under two nursery growing regimes during seven different nursery production periods. New Forests 13:341-356.

Bigras, F.J. 1998. Field performance of containerized black spruce seedlings with root systems damaged by freezing or pruning. New Forests 15:1-9.

Clifton, N.C. 1985. New Zealand radiata pine. New Zealand Forest Service. $31 \mathrm{p}$.

Clutter, J.L., J.C. Fortson, L.V. Pienaar, G.H. Brister y R.L. Bailey. 1983. Timber management: a quantitative approach. Wiley, New York, USA. 333p.

Chacón S., J.M. y H. Manzanilla B. 1988. El sitio experimental "El Poleo" y su comportamiento. In: Memorias del 10응 Aniversario de la Investigación Forestal en el Estado de Chihuahua. SARH INIFAP, Centro de Investigaciones Forestales $y$ Agropecuarias del Estado de Chihuahua, Campo Experimental "Madera". Chihuahua, Chih., México. p:54-67.

García, E. 1988. Modificaciones al Sistema de clasificación climática de Koppen. UNAM. Mexico. 217p.

Dirzo, R. y M.C. García. 1992. Rates of deforestation in Los Tuxtlas, a neotropical area in southeast Mexico. Conservation Biology 6(1):84-90. 
James, R.N.y G.L. Tarlton (eds.) 1990. New approaches to spacing in plantation forestry. Rotorua, New Zealand, 10-14 April 0989. International Union of Forest Research Organizations, New Zealand Forest Research Institute. FRI Bulletin 151. Rotorua, N.Z. 360p.

Mas P., J., G. Naranjo Ch. y H.M. Muñoz F. 1990. Evaluación del desarrollo de once plantaciones forestales establecidas en el municipio de Morelia, Michoacán. SARH INIFAP, Centro de Investigaciones Forestales y Agropecuarias de Michoacán, Campo Experimental Forestal y Agropecuario de Morelia. Folleto Técnico 1. Morelia, Mich. México. 83p.

Maugé, J.P. 1987. Le pin maritime, premier résineux de France. Institute pour le Development Forestiere. París, Francia. 192p.
Mueller-Dumbois, D. y H. Ellemberg. 1974. Aims and methods on vegetation ecology. Wiley, New York, EUA. 547p.

Oliver, C.D. y B.C. Larson. 1996. Forest stand dynamics. Mc Graw Hill, New York, EUA. 467p.

Puttonen, P. 1997. Looking for the "silver bullet"- can one test do it all? New Forests 13:9-27.

Stein, W. 1978. Reforestation evaluation. In:Regenerating Oregon's forests. A guide for the regeneration forester (Cleary, Greaves, Hermann, ed.) School of Forestry, Oregon State University Extension Service. Corvallis, Or. EUA. 287p.

Valdez L., J.R. 1992. Evaluación de la regeneración natural de Pinus hartwegii Lindl., en Zoquiapan, México. Tesis profesional, Universidad Autónoma de Chapingo. Chapingo México. 
